\title{
Frequency-Dependent Substrate Characterization via an Iterative Pole Search Algorithm
}

\author{
Thomas Demeester, Maarten Cauwe and Daniël De Zutter
}

\begin{abstract}
The characterization of frequency-dependent material properties is an important issue in nowadays high-speed interconnect design. This paper presents a practical method to determine the complex permittivity of a substrate material, by combining measurements with simulations. A rational permittivity model is determined by searching for its poles and residues using an iterative optimization method. Its accuracy is verified by comparing coplanar waveguide measurements with simulations based on the new material model.
\end{abstract} tion.

Index Terms-dielectric, permittivity, substrate, characteriza-

\section{INTRODUCTION}

For a correct design of high-speed metallic interconnects, an accurate circuit model is indispensable if the traces are too long for being modeled as lumped resistance-capacitance elements. In this case however, a two-dimensional transmission line model [1] is often sufficient to predict the correct propagation characteristics. The characterization of such a model requires a simulation tool that is able to take into account all relevant phenomena, including low and high frequency losses in the conductors [2], [3], and general losses in the dielectric or semiconductor environment [4], [5]. In order to yield realistic results, these tools need the correct material parameters, that may over the considered frequency range deviate considerably from an often constant data sheet value. The characterization of packaging materials in general has been the topic of many contributions. A good overview of the existing techniques is given in [6] and the references therein. One possible technique is the characterization of substrate materials by means of scattering (S-) parameter measurements. In [7], it is shown how these measurements are used to calculate the propagation factor and attenuation of the fundamental mode, which in turn allows to determine the material parameters, e.g., based on the well-known microstrip design formulas of [8] (which are only approximate in nature).

Section II describes how the problem of determining the dielectric permittivity and loss tangent is cast into a nonlinear optimization problem in which the parameters of a rational fit are obtained using an advanced electromagnetic (EM) simulator. In Section III the resulting material model is verified by using it in a coplanar waveguide (CPW) simulation, comparing the CPW output with measured results.

Manuscript received September 13, 2010; revised December 06, 2010.

T. Demeester and D. De Zutter are with the Department of Information Technology, Ghent University, Ghent, Belgium (e-mail: thomas.demeester@intec.ugent.be, daniel.dezutter@intec.ugent.be).

M. Cauwe is with the Department of Electronics and Information Systems, Ghent University, Ghent, Belgium (e-mail: Maarten.Cauwe@elis.ugent.be)

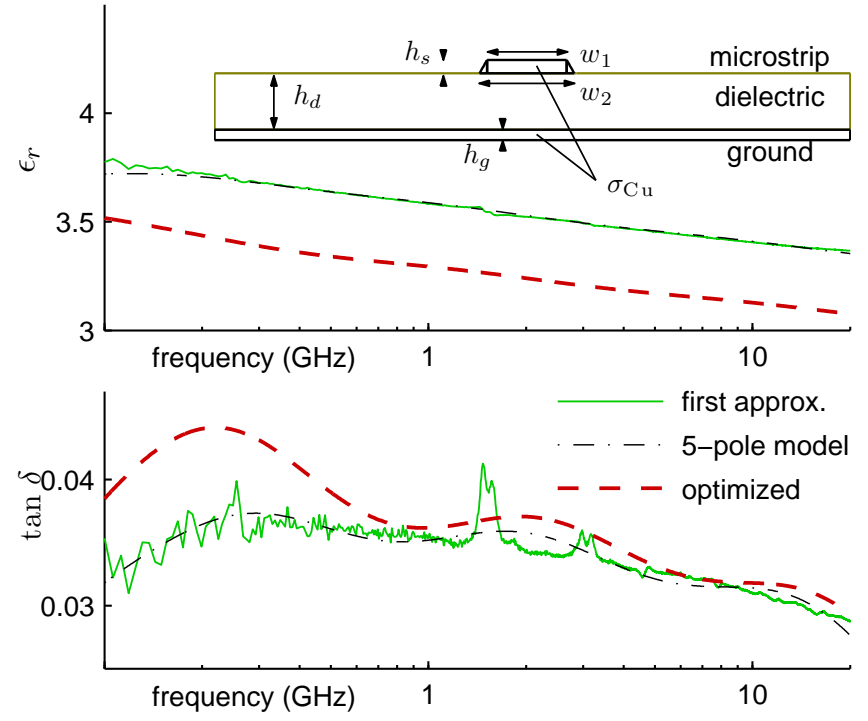

Fig. 1: Inset: simulated microstrip structure, with $\sigma_{\mathrm{Cu}}=$ $5.98 \times 10^{7} \mathrm{~S} / \mathrm{m}$, and $w_{1}=131.8, w_{2}=156.4, h_{s}=21.6$, $h_{d}=93.4$, and $h_{g}=17$ (all in micrometers). Upper and lower graph: $\epsilon_{r}$, respectively, $\tan \delta$ of the substrate, shown for the data obtained by approximate inversion of the measured data, its 5-pole approximation, and the final optimization.

\section{Dielectric MAterial Characterization}

Consider the microstrip, shown in the inset of Fig. 1 and assume an $e^{j \omega t}$ time dependence of the fields, such that the complex permittivity $\epsilon_{c}$ of the substrate can be written

$$
\epsilon_{c}=\epsilon_{0} \epsilon_{r}(1-j \tan \delta)
$$

with $\epsilon_{0}$ the permittivity of free space, $\epsilon_{r}$ the real relative permittivity of the dielectric, and $\tan \delta$ its loss tangent. The metrics used to verify our model for $\epsilon_{c}$, are the attenuation coefficient $\alpha$ and the effective relative permittivity $\epsilon_{r, \text { eff }}$ of the fundamental propagation mode (for which the phase velocity $v$ is given by $v=c / \sqrt{\epsilon_{r, \mathrm{eff}}}$, with $c$ the speed of light in the vacuum). These parameters can be extracted from $\mathrm{S}$-parameter measurements, as part of the multi-line through-reflect-line calibration [7]. The measurements used in this paper were performed using a vector network analyzer in a frequency range from $100 \mathrm{MHz}$ up to $20 \mathrm{GHz}$. The test structures are contacted using coplanar microwave probes on a dedicated printed circuit board probing station. A planar line-reflectmatch (LRM) calibration using a commercial calibration substrate is performed to compensate for internal errors of the network analyzer and the cables, shifting the reference plane 
to the probe tips. A further discussion, concerning the accuracy of these measurements, is outside the scope of this paper (See ref. [7].), and we will hence take $\epsilon_{r, \text { eff }}$ and $\alpha$ obtained from the measurements as a reference for our optimization method.

The permittivity model that we will use consists of a discrete sum of dipole moments,

$$
\epsilon_{c}(s)=\epsilon_{\infty}+\sum_{n=1}^{N} \frac{c_{n}}{s-a_{n}}
$$

with $\epsilon_{\infty}$ a real constant, with the poles $a_{n}$ and residues $c_{n}$ being real, or occurring in complex conjugate pairs, and for stability $\operatorname{Re}\left(a_{n}\right)<0$. This model, for $s=j 2 \pi f$, automatically satisfies the Kramers-Kronig relations, which impose a necessary condition for a physically correct model, due to causality requirements [9]. The proposed procedure consists of the following steps:

1) Using the vector fitting technique [10], initial values of $\epsilon_{\infty}, c_{n}$ and $a_{n}$ are determined based on the measured data and the design formulas of [8] (eq. 3, pp. 1611) and [7] (eq. 5-9, pp. 652). These formulas only approximate the actual EM behavior, e.g., conductor losses are not taken into account. The number $N$ of poles can be kept low (e.g., 3 to 5), in view of the limited variation of $\epsilon_{c}$ in the considered frequency range (Fig. 1).

2) The initial values are refined using the aforementioned EM tool. This tool does account for conductor losses and all relevant high frequency EM phenomena. Feeding the EM simulator with $\epsilon_{c}$ (2), using the initial guess of step 1, will lead to simulated results for $\epsilon_{r \text {,eff }}$ and $\alpha$ that differ from the measured ones. Hence, better guesses are needed. To obtain these guesses, we use a Particle Swarm Optimization (PSO) algorithm [11]. Other approaches are of course possible [12].

Remark that the used EM simulator, from [5], does account for conductor losses as the copper lines have a finite crosssection with a large but finite conductivity $\sigma$. However, $\sigma$ is kept constant while $\epsilon_{c}$ varies until the measured and simulated $\epsilon_{r, \text { eff }}$ and attenuation $\alpha$ coincide.

The PSO algorithm needs a cost function $\mathcal{F}$, to be minimized iteratively. To this end, we introduce the complex wave number $\beta$ with $\operatorname{Re} \beta=\omega \sqrt{\epsilon_{r, \mathrm{eff}}} / c$ and $\operatorname{Im} \beta=$ $-1000 \alpha /\left(20 \log _{10} e\right)$ (the proportionality factor is merely to express $\alpha$ in $\mathrm{dB} / \mathrm{mm}$, as used in this paper). With $\beta_{\text {sim }}$ the simulation result, $\beta_{\text {ref }}$ the measurement value, and

$$
\Delta \beta \stackrel{\text { def }}{=}\left|\beta_{\text {sim }}-\beta_{\text {ref }}\right|
$$

$\mathcal{F}$ is defined as

$$
\mathcal{F} \stackrel{\text { def }}{=} \frac{\sqrt{\left\langle(\operatorname{Re} \Delta \beta)^{2}\right\rangle}}{\left\langle\left|\operatorname{Re} \beta_{\text {ref }}\right|\right\rangle}+\frac{\sqrt{\left\langle(\operatorname{Im} \Delta \beta)^{2}\right\rangle}}{\left\langle\left|\operatorname{Im} \beta_{\text {ref }}\right|\right\rangle} .
$$

The angular brackets $\langle$.$\rangle denote the average over the consid-$ ered frequency band. The denominators in (4) normalize the real and imaginary part of $\Delta \beta$, to give a comparable weight to $\operatorname{Re} \beta$ (and hence $\epsilon_{r, \text { eff }}$ ) and to $\operatorname{Im} \beta$ (related to $\alpha$ ). With the limits of the search space ranging in each dimension over a hundred percent of the initial values (always taking into account the limitations for a causal model), a good result is found within about 10 minutes (for each simulation taking around 5 seconds) on a standard office pc.

In Fig. 1, the substrate material parameters are shown as obtained by using the procedure outline above for the microstrip structure. A first dataset is obtained by inversion of the measured data $\epsilon_{r, \text { eff }}$ and $\alpha$, using the mentioned formulas of [8] and [7]. The figure also shows the 5-pole model fit to this first dataset, and the final optimized material parameters.

\section{VALIDATION}

Although the optimized $\epsilon_{r}$ and $\tan \delta$ values shown in Fig. 1 lead to an excellent agreement between measured and simulated data for the $\epsilon_{r, \text { eff }}$ and attenuation $\alpha$ of the signal propagated along the microstrip line, this is after all not surprising as our optimization procedure enforces this fit. In order to verify if the obtained material parameters are indeed real physical parameters, the following independent validation is performed. We manufacture a completely different waveguide structure, in casu a coplanar waveguide (CPW), on the very same substrate as the microstrip line, i.e., using exactly the same material. The propagation characteristics of this CPW ( $\epsilon_{r \text {,eff }}$ and attenuation $\alpha$ ) are determined by S-parameter measurements and also simulated using the advanced EM-tool used before. No optimization takes place. If measured and simulated data coincide, this can be considered a strong indication that the method we propose to determine the material parameters is valid. Let us first look at some details of the test board. The test board was manufactured in-house using standard printed circuit board processing: lamination, via drilling, plating and structuring, on an Isofoil 160i substrate from CircuitFoil. The patterning of the test structures was done by wet etching of the copper. A picture of the board, containing the microstrip lines and the CPW's, is shown in Fig. 2. The cross-section of such a CPW structure is shown in Fig. 3, displaying the simulated geometry on top of a photographed cross-section. Note that the reference planes are the conductors on both sides of the signal line, and the plane underneath the substrate is standing free. The triangles at the conductor sides are used to ensure a good modeling of the non-rectangular conductors, that are a result of the wet etching procedure. The dimensions indicated in Fig. 3, are an average of three to five cross-section measurements on five different lines that were used for the Sparameter measurements (see Fig. 2). In Fig. 4, $\epsilon_{r \text {,eff }}$ and $\alpha$ are shown from the measurements, and compared to the simulation results, with a very good correspondence.

Despite these promising results, the authors are aware of a few factors, which are not obvious to control, but that might have influenced the results. First, as already mentioned, the accuracy of the determined material parameters is limited by the accuracy of the S-parameter measurements of the interconnects. However, as all measurements were performed on the same board, with the same setup and the same calibration procedures, one can assume that any remaining systematical measurement inaccuracies have little or no influence on the final results shown in Fig. 4. Furthermore, results are sensitive to geometry uncertainties in both structures, as these 


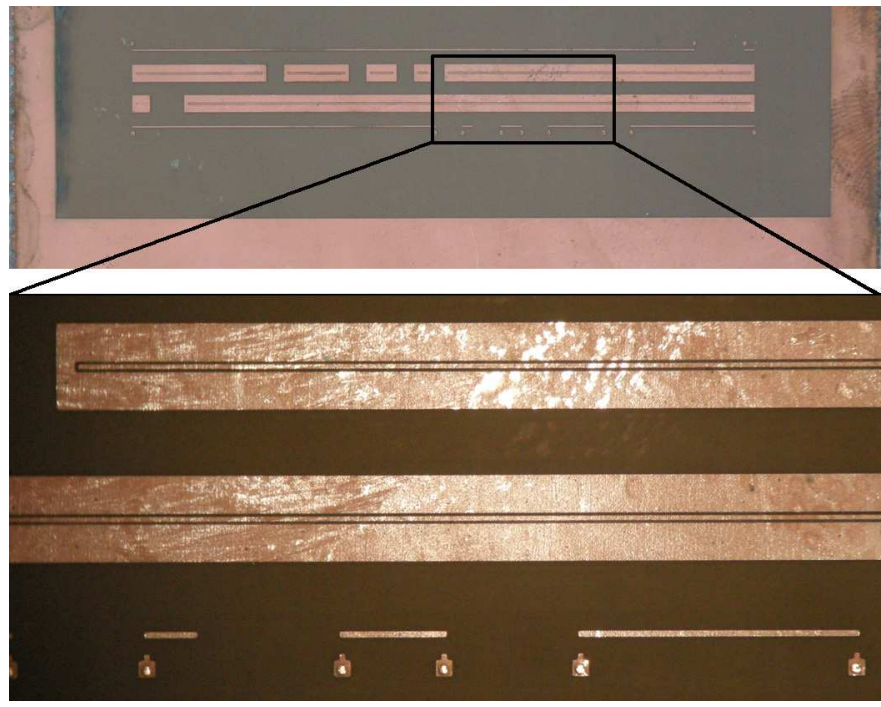

Fig. 2: Test board containing the microstrip lines used for material characterization and the CPW test lines. Upper picture, entire board; lower picture, detail.

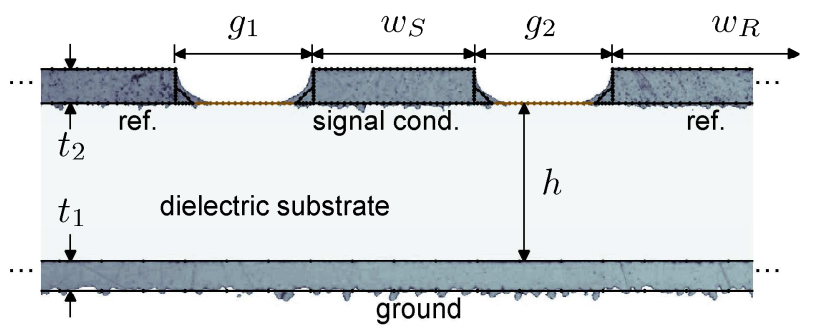

Fig. 3: Coplanar waveguide, with the simulated geometry on top of a cross-section photograph. The dimensions (all in micrometers) are $g_{1}=81.0, g_{2}=81.3, w_{S}=94.3$, $w_{R}=870, t_{1}=17.0, t_{2}=20.3, h=92.7$. All conductors are copper $\left(\sigma_{\mathrm{Cu}}=5.98 \times 10^{7} \mathrm{~S} / \mathrm{m}\right)$.

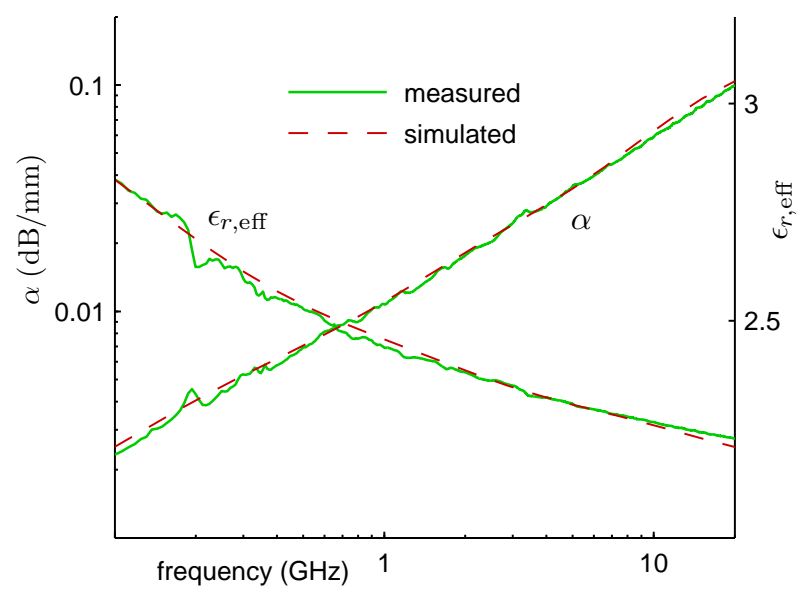

Fig. 4: Effective relative permittivity and attenuation constant for the coplanar waveguide of Fig. 3.

influence the effective permittivity. The conductor width of the microstrip, and the gaps and conductor shape of the CPW are the most important factors. A detailed sensitivity analysis remains however a topic of further research. Finally, the better the simulation software, the better the results will be. The authors believe their software is state-of-the-art in high-frequency conductor modeling, but the influence of, for example, the conductor roughness (clearly visible in Fig. 3) is not accounted for, and might also influence the accuracy of the results.

\section{CONCLUSION}

The technique proposed in this paper can be used to estimate frequency-dependent material parameters in interconnect structures. The optimal values are those that allow matching carefully simulated characteristics with measurements. In the described experiment, the parameters of a rational fit for the dielectric constant of a microstrip substrate were optimized iteratively. The resulting material model was used in a coplanar waveguide simulation, and comparing the resulting propagation characteristics with measurements allowed to verify the validity of the method.

Although the results are promising, some drawbacks are mentioned. The accuracy of the result is limited by the correctness of the simulations, the measurements, and the sensitivity of the permittivity to small geometry variations.

\section{REFERENCES}

[1] F. Olyslager, Electromagnetic Waveguides and Transmission Lines, ser. Oxford Engineering Science Series 51. Oxford, U.K.: Oxford University Press Inc., 1999.

[2] K. Coperich, A. Ruehli, and A. Cangellaris, "Enhanced skin effect for partial-element equivalent-circuit (PEEC) models," IEEE Trans. Microw. Theory Tech., vol. 48, no. 9, pp. 1435-1442, Sep. 2000.

[3] D. De Zutter and L. Knockaert, "Skin effect modeling based on a differential surface admittance operator," IEEE Trans. Microw. Theory Tech., vol. 53, no. 8, pp. 2526-2538, Aug. 2005.

[4] G. Plaza, R. Marques, and F. Medina, "Quasi-TM MoL/MoM approach for computing the transmission-line parameters of lossy lines," IEEE Trans. Microw. Theory Tech., vol. 54, no. 1, pp. 198-209, Jan. 2006.

[5] T. Demeester and D. De Zutter, "Quasi-TM transmission line parameters of coupled lossy lines based on the Dirichlet to Neumann boundary operator," IEEE Trans. Microw. Theory Tech., vol. 56, no. 7, pp. 16491660, Jul. 2008.

[6] J. Baker-Jarvis and M. D. Janezic, "Dielectric and conductor-loss characterization and measurements on electronic packaging materials," Nat. Inst. Standards Technol., Gaithersburg, MD, Tech. Rep. 1520, Jul. 2001.

[7] M. Cauwe and J. De Baets, "Broadband material parameter characterization for practical high-speed interconnects on printed circuit board,' IEEE Trans. Adv. Packag., vol. 31, no. 3, pp. 649 -656, Aug. 2008.

[8] I. Bahl and R. Garg, "Simple and accurate formulas for a microstrip with finite strip thickness," vol. 65, no. 11, pp. 1611 - 1612, Nov. 1977.

[9] P. Triverio, S. Grivet-Talocia, M. Nakhla, F. Canavero, and R. Achar, "Stability, causality, and passivity in electrical interconnect models," IEEE Trans. Adv. Packag., vol. 30, no. 4, pp. 795 -808, Nov. 2007.

[10] B. Gustavsen and A. Semlyen, "Rational approximation of frequency domain responses by vector fitting," IEEE Trans. Power Del., vol. 14, no. 3, pp. $1052-1061$, Jul. 1999.

[11] J. Kennedy and R. Eberhart, "Particle swarm optimization," in Neural Networks, 1995. Proceedings., IEEE International Conference on, vol. 4, nov/dec 1995, pp. $1942-1948$.

[12] I. Couckuyt, F. Declercq, T. Dhaene, H. Rogier, and L. Knockaert, "Surrogate-based infill optimization applied to electromagnetic problems," International Journal of RF and Microwave Computer-Aided Engineering (Wiley RFMiCAE), Special Issue on Advances in Design Optimization of Microwave/RF Circuits and Systems, vol. 20, no. 5, pp. $492-501$, Sep. 2010. 